Microchannels and Minichannels - 2004

June 17-19, 2004, Rochester, New York, USA

Copyright (C) 2004 by ASME

ICMM2004 - XXXX

\title{
VALIDATION OF A SECOND-ORDER SLIP MODEL FOR TRANSITION-REGIME, GASEOUS FLOWS
}

\author{
Nicolas G. Hadjiconstantinou \\ Mechanical Engineering Department, Massachusetts Institute of Technology, Cambridge, MA USA, 02139 ngh@mit.edu
}

\begin{abstract}
We discuss and validate a recently proposed secondorder slip model for dilute gas flows. Our discussion focuses on the importance of quantitatively accounting for the effect of Knudsen layers close to the walls. This is important, not only for obtaining an accurate slip model but also for interpreting the results of the latter since in transition-regime flows the Knudsen layers penetrate large parts of the flow. Our extensive validation illustrates the above points by comparing direct Monte Carlo solutions to the slip model predictions for an unsteady flow. Excellent agreement is found between simulation and the slip model predictions up to $\mathrm{Kn}=0.4$, for both the velocity profile and stress at the wall. This demonstrates that the proposed second-order slip model reliably describes arbitrary flowfields (and related stress fields) in a predictive manner at least up to $\mathrm{Kn}=0.4$ for both steady and transient problems.
\end{abstract}

\section{INTRODUCTION}

Second-order slip models can extend the range of applicability of the Navier-Stokes description beyond $\mathrm{Kn} \cong 0.1$ where the accuracy of first-order slip models begins to deteriorate. Given the simplicity and negligible cost of Navier-Stokes solutions compared to molecular simulations, accurate second-order slip models are very desirable. In a recent paper, a second-order slip model for a hard sphere gas was presented [1]. This model is different from previous approaches in two important aspects. First, it is based on a solution of the Boltzmann equation [4] and has no adjustable/fitted parameters. Second, it accounts in a rigorous manner for the deviation from Navier-Stokes behavior in the near-wall regions known as Knudsen layers [4]. This, in fact, holds the key to developing a successful second-order slip model: Our discussion along these lines will illustrate why approaches which do not take this into account are destined to fail. The slip model discussed here has already been shown to be in excellent agreement with DSMC simulations of pressure driven flow for $\mathrm{Kn} \leq 0.4$ [1].

In this paper we apply this model to the description of oscillatory Couette flows. The results obtained are compared to direct Monte Carlo simulations. The objectives of this investigation are numerous: First, it serves as an independent validation of the proposed slip model. It will be seen that the second-order slip model provides solutions that are in good agreement with molecular simulations for both the velocity field and stress at the wall for $\mathrm{Kn} \leq 0.4$. Second, the transient nature of the flow provides a more demanding test of the slip-flow model since the latter was originally derived under the assumption of steady flow. Our results suggest that the slip model remains robust even at oscillation frequencies that approach (but remain much smaller than) the molecular collision frequency. Third, this paper serves to illustrate the effect of the Knudsen layers on the flow and how this is taken into account by the slip model. Finally, our investigation is motivated by the practical interest in these flows [2,3].

\section{SECOND-ORDER SLIP MODEL}

The recently proposed model [1] predicts that the slip velocity $\left.u\right|_{\text {wall }}-u_{\mathrm{w}}$ in a one-dimensional flow of a hard sphere gas is given by 


$$
\left.u\right|_{\text {wall }}-u_{\mathrm{w}}=\left.\alpha \lambda \frac{\partial u}{\partial \eta}\right|_{\text {wall }}-\left.\beta \lambda^{2} \frac{\partial^{2} u}{\partial \eta^{2}}\right|_{\text {wall }}
$$

where $\alpha=1.11, \beta=0.61,\left.\right|_{\text {wall }}$ denotes quantities evaluated at the wall, $u_{\mathrm{w}}$ is the wall velocity, and $\eta$ is the normal to the wall pointing into the gas. However, care needs to be taken when using second-order models; as pointed out by Cercignani [4], second-order slip models predict the flowfield outside the Knudsen layers (thin layers close to walls where strong non-equilibrium effects are important) whose thickness is of the order of one mean free path. Inside the Knudsen layers the flowfield deviates from the slip-corrected Navier-Stokes prediction to the extent that the average velocity differs from the Navier-Stokes result to $O\left(\mathrm{Kn}^{2}\right)$. As a result, when a second-order slip model is used, estimates of the mean flow velocity need to be corrected for the effects of the Knudsen layers. According to the slip model, in a one-dimensional channel flow, the mean flow velocity is given by [1]

$$
\bar{u}=\frac{1}{L} \int_{-L / 2}^{L / 2}\left[u+\xi \lambda^{2} \frac{\partial^{2} u}{\partial y^{2}}\right] d y
$$

where $\xi \cong 0.3, y$ is the spatial coordinate and $L$ is the distance between the walls.

A consequence of equation (2) is that experiments performed to measure the second-order slip coefficient through some measure of the mean flow, in fact measure an "effective" second-order slip coefficient that is different from $\beta$. For example, typical experiments measure the flow rate per unit depth $(\bar{u} L)$ in pressure-driven (Poiseuille) flow; on the other hand, it follows from equation (2) that these experiments measure an effective second-order slip coefficient which is equal to $\beta-\xi$ (recall that the Poiseulle flow profile has a constant curvature - see also [1]). This explains recent experimental measurements [8] which find a second-order slip coefficient of approximately $0.25 \pm 0.1$; this is, in fact, in good agreement with the theoretical prediction of $0.61-0.3=0.31$.

\section{VALIDATION PROBLEM DESCRIPTION}

We consider a dilute gas between two infinitely long, smooth, fully accommodating walls located at $y=0$ and $y=L$ respectively. One of the walls $(y=L)$ moves sinusoidally in time with amplitude $U$ and with frequency $\omega$ which is expressed here as $u_{\mathrm{w}}=U \operatorname{Im}(\exp (i \omega t))$. The equation governing the gas motion is

$$
\frac{\partial u}{\partial t}=v \frac{\partial^{2} u}{\partial y^{2}}
$$

where $v$ is the gas kinematic viscosity. The solution of this equation subject to the second-order slip condition (1) is given by

$$
u=\operatorname{Im}\left(A \sinh \zeta \frac{y}{L}+B \cosh \zeta \frac{y}{L}\right)
$$

where

$$
\begin{aligned}
& A=\frac{U \exp (i \omega t)}{C+\frac{\alpha \mathrm{Kn} \zeta}{1+\beta \zeta^{2} \mathrm{Kn}^{2}} D} \\
& B=\frac{\alpha \mathrm{Kn} \zeta}{1+\beta \zeta^{2} \mathrm{Kn}^{2}} A \\
& C=\sinh \zeta+\alpha \mathrm{Kn} \zeta \cosh \zeta+\beta \mathrm{Kn}^{2} \zeta^{2} \sinh \zeta \\
& D=\cosh \zeta+\alpha \mathrm{Kn} \zeta \sinh \zeta+\beta \mathrm{Kn}^{2} \zeta^{2} \cosh \zeta
\end{aligned}
$$

$\zeta=\sqrt{i} \vartheta$, and $\vartheta=\sqrt{\omega L^{2} / v}$ is the Stokes number [2]. Implicit in this solution and the use of a slip model is the assumption that the stress is given by the linear constitutive law $\tau_{i j}=\mu\left(\partial u_{i} / \partial x_{j}+\partial u_{j} / \partial x_{i}\right)$ throughout the domain. Therefore one critical test of any slip model is whether it captures not only the velocity but also the stress field with no extra adjustable parameters.

\section{COMPARISON WITH DSMC SIMULATIONS}

We performed direct Monte Carlo simulations [5] to test the validity of the above result and hence the ability of the second-order slip model to describe arbitrary flows with no adjustable parameters. Our simulations used $10^{5}$ particles and 25 collision cells. The simulation timestep was taken to be $2.09 \times 10^{-11} \mathrm{~s}$ which is slightly longer than one tenth of the molecular collision time $1 / \varepsilon$. Typically $2 \times 10^{6}$ timesteps were performed; the velocity field was sampled after $0.5 \times 10^{6}$ timesteps allowing the simulation to reach steady state and leading to a sample size of approximately 2000 oscillation periods. Otherwise, standard DSMC procedures $[5,6]$ were used.

The Knudsen number was varied between $0.1 \leq \mathrm{Kn} \leq 0.4$ and the frequency was chosen to be small compared to the collision frequency but also sufficiently high $(\vartheta \sim 1)$ such that the flow was not in the quasistatic regime $(\vartheta<<1)$ where linear velocity profiles are observed. The wall velocity amplitude, $U$, was set to $0.1 \sqrt{\gamma R T}$; this choice represents a reasonable balance between the requirement of high speeds for good signal to noise ratio [7] and low speeds for negligible compressibility and viscous heating effects.

The majority of the comparisons shown here were performed at a fixed time $(t=\mathrm{T} / 2$ where $\mathrm{T}=2 \pi / \omega$ is the 
oscillation period). This time was chosen because at this time the effects of the Knudsen layers are more clearly visible. In general, the level of agreement found was independent of the time, as one would normally expect. At the end of the section we provide a sample comparison at $t=T / 4$ for completeness.

Figure 1 shows a comparison between the two solutions for $\mathrm{Kn}=0.1, \quad \vartheta=5 / \sqrt{2}$ at time $t=\mathrm{T} / 2$. The agreement between the two solutions is excellent.

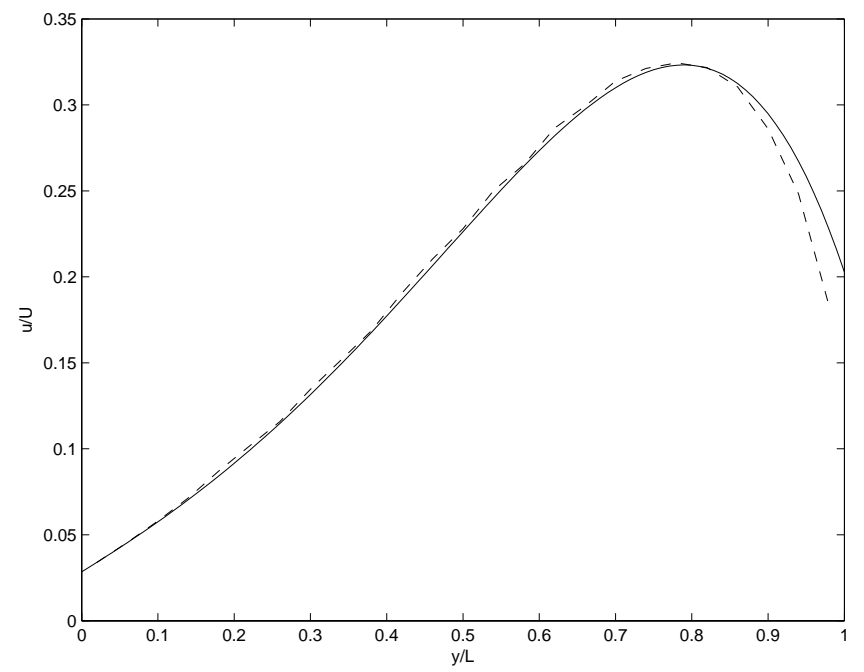

Figure 1: Comparison between Equation (4) (solid line) and DSMC results (dashed line) for $\mathrm{Kn}=0.1$, $\vartheta=5 / \sqrt{2}$.

This figure also clearly shows the effect of the Knudsen layers close to the walls. The slip-corrected Navier-Stokes solution deviates from the DSMC result within the Knudsen layers, which in this case are about $L / 10$ thick. The discrepancy due to the Knudsen layer depends on the local curvature in the velocity-field; this is clearly demonstrated in Figure 1. Its effect on the average velocity, to second order in $\mathrm{Kn}$, can be captured by the proposed model (see Equation (2)).

Figure 2 shows a comparison of the slip model and DSMC results for $\mathrm{Kn}=0.2, \vartheta=2.5 / \sqrt{2}$. The effect of the Knudsen layers becomes more pronounced but the agreement in the remainder of the domain remains very good. At $\mathrm{Kn}=0.4, \vartheta=1.25 / \sqrt{2}$ the effect of the Knudsen layers is very significant. At this Knudsen number the domain size is only 2.5 mean free paths wide. Therefore it is possible that the discrepancy in Figure 3 is because the Knudsen layers have penetrated to the middle of the domain: Figures 1 and 2 suggest that the Knudsen layer thickness exceeds one mean free path and thus the latter explanation is possible. In fact, the average velocity given by Equation (2) is in excellent agreement with the DSMC result (see Table 1) all the way to $\mathrm{Kn}=0.4$, which also suggests that the discrepancy observed in Figure 3 is due to the Knudsen layers rather than systematic error.
In Table 2 we compare the magnitude of the stress at the two walls predicted by the slip-corrected Navier-Stokes description and DSMC. This is an important test, not only because the shear stress is a quantity of interest for this problem, but because it tests the very basis of a slip model, namely that the linear constitutive relation is valid throughout the physical domain. In fact, since the nonequilibrium effects responsible for the failure of the NavierStokes description originate from the system walls, capturing the shear stress at the wall can be considered as one of the most stringent tests of this model.

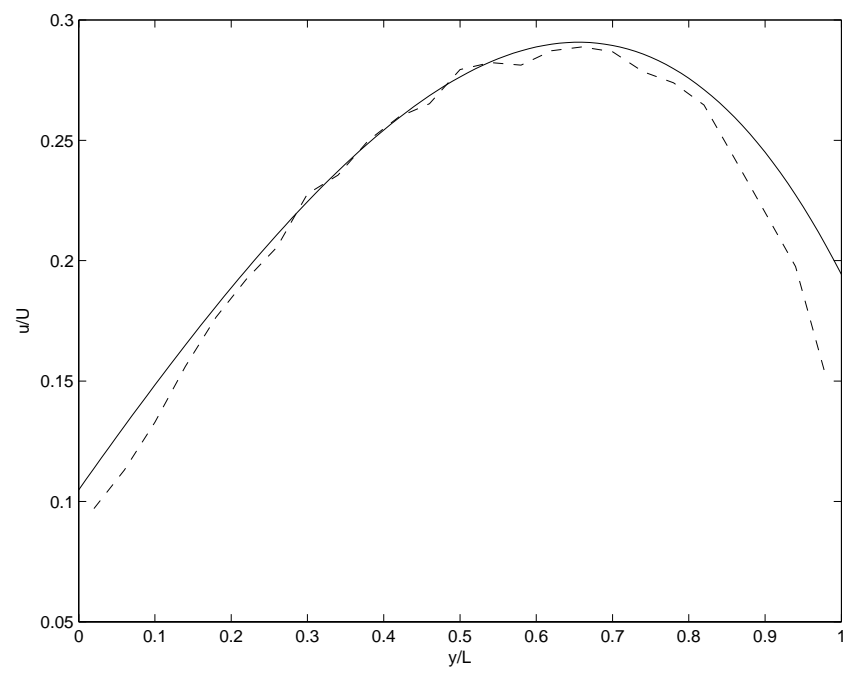

Figure 2: Comparison between Equation (4) (solid line) and DSMC results (dashed line) for $\mathrm{Kn}=0.2, \vartheta=2.5 / \sqrt{2}$.

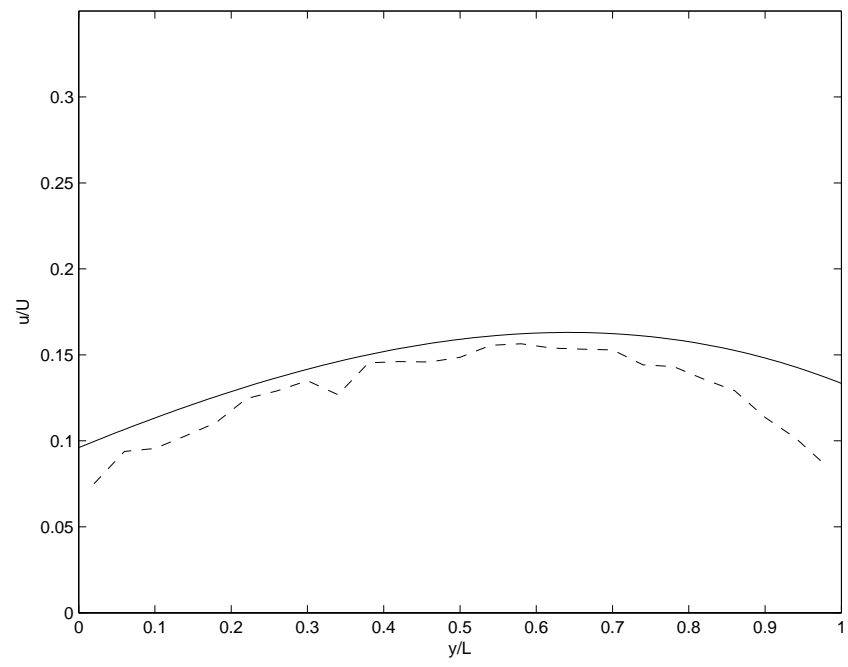

Figure 3: Comparison between Equation (4) (solid line) and DSMC results (dashed line) for $\mathrm{Kn}=0.4, \vartheta=1.25 / \sqrt{2}$.

The non-dimensional shear stress $\widehat{\tau}_{w}$ at the wall is given by $\tau_{w} /(\mu U / L)$ where $\tau_{w}$ is the shear stress at the wall. All 
values are given at $t=\mathrm{T} / 2$. This table shows that the error in the stress is very small even for $\mathrm{Kn}=0.4$.

Table 1: Average velocity at $t=\mathrm{T} / 2$

\begin{tabular}{|c|c|c|}
\hline $\mathrm{Kn}$ & $\bar{u}^{N S}$ & $\bar{u}^{\text {DSMC }}$ \\
\hline 0.1 & 0.221 & 0.226 \\
\hline 0.2 & 0.196 & 0.200 \\
\hline 0.4 & 0.127 & 0.128 \\
\hline
\end{tabular}

Table 2: Normalized shear stress at the walls at $t=\mathrm{T} / 2$

\begin{tabular}{|l|c|c|c|c|}
\hline $\mathrm{Kn}$ & $\left|\bar{\tau}_{w}\right|_{y=0}^{N S}$ & $\left|\bar{\tau}_{w}\right|_{y=0}^{D S M C}$ & $\left|\bar{\tau}_{w}\right|_{y=L}^{N S}$ & $\left|\bar{\tau}_{w}\right|_{y=L}^{D S M C}$ \\
\hline 0.1 & 0.27 & $0.26 \pm 0.04$ & 1.33 & $1.38 \pm 0.04$ \\
\hline 0.2 & 0.45 & $0.44 \pm 0.02$ & 0.62 & $0.65 \pm 0.02$ \\
\hline 0.4 & 0.18 & $0.175 \pm 0.01$ & 0.175 & $0.19 \pm 0.01$ \\
\hline
\end{tabular}

In Figure 4 we show the results of a comparison at a time other than $t=\mathrm{T} / 2$. In particular, this figure shows a comparison for $\vartheta=2.5 / \sqrt{2}$ and $\mathrm{Kn}=0.2$ at $t=\mathrm{T} / 4$. The agreement is excellent similarly to the comparisons at $t=\mathrm{T} / 2$. It is for this reason that the majority of comparisons was limited to one time, namely $t=\mathrm{T} / 2$. The Navier Stokes (DSMC) value of $\left|\hat{\tau}_{w}\right|$ is $0.34(0.38 \pm 0.03)$ at $y=0$ and $1.08(1.04 \pm 0.03)$ at $y=L$.

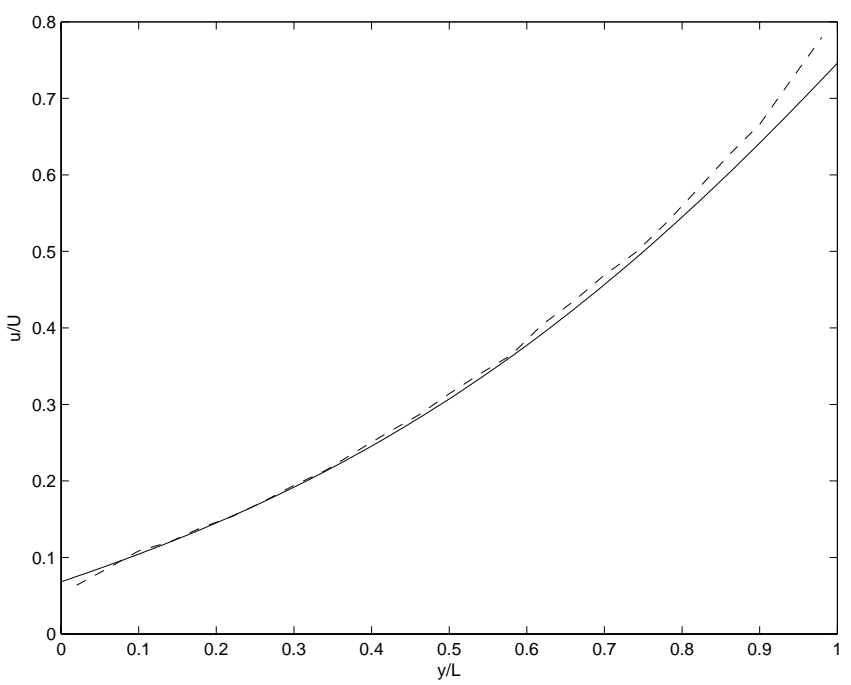

Figure 4: Comparison between Equation (4) (solid line) and DSMC results (dashed line) for $\mathrm{Kn}=0.2$ at $t=\mathrm{T} / 4$, $\vartheta=2.5 / \sqrt{2}$.

\section{CONCLUSIONS}

We have presented a comparison between a recently proposed second-order slip model and DSMC simulations for oscillatory Couette flows. The particular problem was chosen due to its practical importance but also because it provides flowfields that are quantitatively different from the steady, pressure-driven flow for which the second-order model has been tested previously. The transient nature of the flow also provides a more stringent test of the model.

Our results show that the slip model provides very good Navier-Stokes approximations to the actual flow. By this we mean that the Navier-Stokes solution is accurate outside the Knudsen layers, while inside the latter the Navier-Stokes solution provides a baseline from which the Knudsen layer contribution can be subtracted. The slip-model also predicts the stress on the wall accurately with no adjustable parameters. It also appears that the steady assumption under which the slip model was derived can be lifted, presumably as long as the flow timescale is long compared to the molecular collision time.

\section{ACKNOWLEDGEMENTS}

The author wishes to thank Dr. M. Gallis for helpful discussions.

\section{NOMENCLATURE}

$\begin{aligned} i & =\sqrt{-1} \\ k & =\text { Boltzmann's constant } \\ \mathrm{Kn} & =\text { Knudsen number }=\lambda / L \\ L & =\text { Distance between walls } \\ m & =\text { Molecular mass } \\ R & =\text { Gas constant }=k / m \\ T & =\text { Temperature } \\ \mathrm{T} & =\text { Oscillation period } \\ u & =\text { Gas velocity } \\ \bar{u} & =\text { Bulk velocity } \\ \bar{u}^{N S} & =\text { Average velocity calculated using slip-corrected } \\ \bar{u}^{D S M C} & =\text { Avavier Stokes equation } \\ y & =\text { Solution velocity calculated from DSMC } \\ & \end{aligned}$

\section{Greek Symbols}

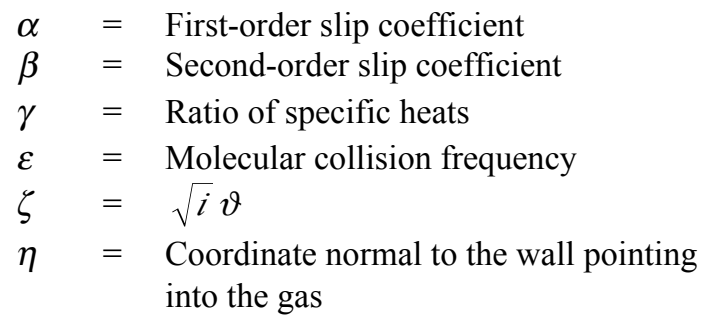




$$
\begin{aligned}
\vartheta & =\sqrt{\omega L^{2} / v}=\text { Stokes number } \\
\lambda & =\text { Molecular mean free path } \\
\mu & =\text { Coefficient of viscosity } \\
v & =\mu / \rho=\text { Kinematic viscosity } \\
\xi & =0.296[1] \\
\rho & =\text { Mass density } \\
\tau_{w} & =\text { Shear stress at the wall } \\
\hat{\tau}_{w} & =\tau_{w} /(\mu U / L) \\
\omega & =\text { Oscillation frequency }
\end{aligned}
$$

\section{REFERENCES}

[1] Hadjiconstantinou, N.G., 2003, "Comment on Cercignani's second-order slip coefficient," Physics of Fluids, 15(8), pp. 2352-2354.

[2] Bahukudumbi, P, Park, J.H and Beskok, A. "A Unified Engineering Model for Steady and Unsteady Shear-Driven Gas Microflows," Microscale Thermophysical Engineering, 7, 291-315, 2003.

[3] Park, J.H., Bahukudumbi, P. and Beskok, A., "Rarefaction Effects on Shear Driven Oscillatory Gas Flows: A DSMC Study in the Entire Knudsen Regime," Physics of Fluids, 16, 317-330, 2004.

[4] Cercignani, C., 1988, The Boltzmann equation and its applications, Springer-Verlag, New York.

[5] Bird, G.A., 1994, Molecular Gas Dynamics and the Direct Simulation of Gas Flows, Clarendon Press, Oxford.

[6] Hadjiconstantinou, N.G., 2002, "Sound wave propagation in transition-regime micro- and nanochannels," Physics of Fluids, 14(2), pp. 802-809.

[7] Hadjiconstantinou, N.G., Garcia, A.L., Bazant, M.Z. and He, G., 2003, "Statistical error in particle simulations of hydrodynamic phenomena," J. Comp. Phys., 187(1), pp. 274-297.

[8] Maurer, J., Tabeling, P., Joseph, P. and Willaime, H., 2003, "Second-order slip laws in microchannels for helium and nitrogen," Physics of Fluids, 15, 2613-2621. 